\title{
Pygmi: Guided Microscope Control Interface with Workflow Manager
}

\author{
$\underline{\text { Mirna Lerotic }}^{1}$, Ian McNulty ${ }^{2}$ and Martin V. Holt ${ }^{2}$ \\ 1. 2nd Look Consulting, 1702, 17/F, Tung Hip Commercial Building, 248 Des Voeux Road, Hong Kong \\ 2. Center for Nanoscale Materials, Argonne National Laboratory, Argonne, Illinois 60439, USA \\ *mirna@2ndlookconsulting.com
}

Due in large part to the recent development of high-brightness sources and nanoscale focusing optics in the multi-keV regime, x-ray microscopy is playing an increasingly broad role in imaging and characterization across many different fields of science. With this increase in popularity, access to synchrotron x-ray microscopes is getting more and more competitive. Typically, users get allocated a few days for theirs experiments, part of which must be spent on learning how to operate the microscope, collect and visualize data. Minimizing users training time, while removing lost time due to operator confusion and resolving problems, ultimately leads to more successful experiments and more efficient use of allocated time.

In this paper we describe Pygmi (PYthon Guided Microscope control Interface), a computer program designed for task-oriented user microscope control. Pygmi was developed for CNM/APS Hard X-ray Nanoprobe [1] to organize microscope operation, beamline alignment, and data visualization tasks typically split among several different software packages into a single application. This simplifies microscope operation by guiding the user through task-based workflows and providing easy access to equipment and detectors. Among other features, Pygmi provides individual help pages for each detector and equipment, a task-based workflow manager, and personalized log books providing interactive access to user-designed scans, workflows, and configurations.

Pygmi is written entirely in Python, uses PyQt for its graphical user interface (UI), and PyEpics [2] to communicate with Epics Control System [3]. This maintains the microscope user interface as a distinct software control / organizational layer that modularly interacts with an externally hosted EPICS layer the EPICS layer is responsible for lower level device support, motion control, and scanning, greatly simplifying implementation of both higher level and lower level roles. Although the program was developed for Hard X-ray Nanoprobe, it can easily be used for other instruments which use Epics. Moreover, due to its inherently modular design Pygmi can be adapted to use a different device control and scanning system, such as spec.

The main Pygmi UI, shown in Figure 1, uses a palette-based approach with interacting windows. Epics motors and devices are defined through Equipment Setup Tab of the Settings panel and can be viewed with the Equipment Browser through which users can access individual positioner help files, launch positioner Control Window, or add a positioner to the Equipment Monitor. Similarly, detectors are defined through the Detector Setup Tab. Depending on the detector type, various current information can be displayed through Detector Browser. When performing scans, scan settings can be defined via Scan Setup Window, or loaded from the current Epics scan records. Pygmi also allows users to browse through scan history by looking at the last ten acquired scans, or by dragging positioner icons in order to bring up the last scan the positioner was used in.

Commons tasks on the microscope range from beamline maintenance such as microscope alignment to 
user experiments, and often they require access to specific equipment and step-by-step execution. Pygmi tasks can be used for both beamline control and to run user experiments. Moreover, they are designed to guide the user through different required steps. After selecting a task, UI is populated with relevant equipment and the user is presented with a task workflow manager. Workflow steps include notes, positioner setpoints (used to move motors and devices to predefined reference points), and scans. Such workflows can be either run automatically, without any interaction required from the user, or each step can be loaded and run separately. Deselecting a task hides the workflow manager and closes the control windows which are associated with the task in order to reduce clutter in the user space. By guiding users through tasks and providing easy access to equipment and detectors, Pygmi shortens training time for new users and makes running experiments more efficient, successful, and streamlined [4].

\section{References:}

[1] Winarski RP, Holt MV, Rose V, et al. A hard X-ray nanoprobe beamline for nanoscale microscopy. Journal of Synchrotron Radiation. 2012;19(Pt 6):1056-1060. doi:10.1107/S0909049512036783.

[2] http://pyepics.github.io/pyepics/.

[3] https://epics.anl.gov/.

[4] This work was performed in part at the Center for Nanoscale Materials, a U.S. Department of Energy Office of Science User Facility, and supported by the U.S. Department of Energy, Office of Science, under Contract No. DE-AC02-06CH11357.

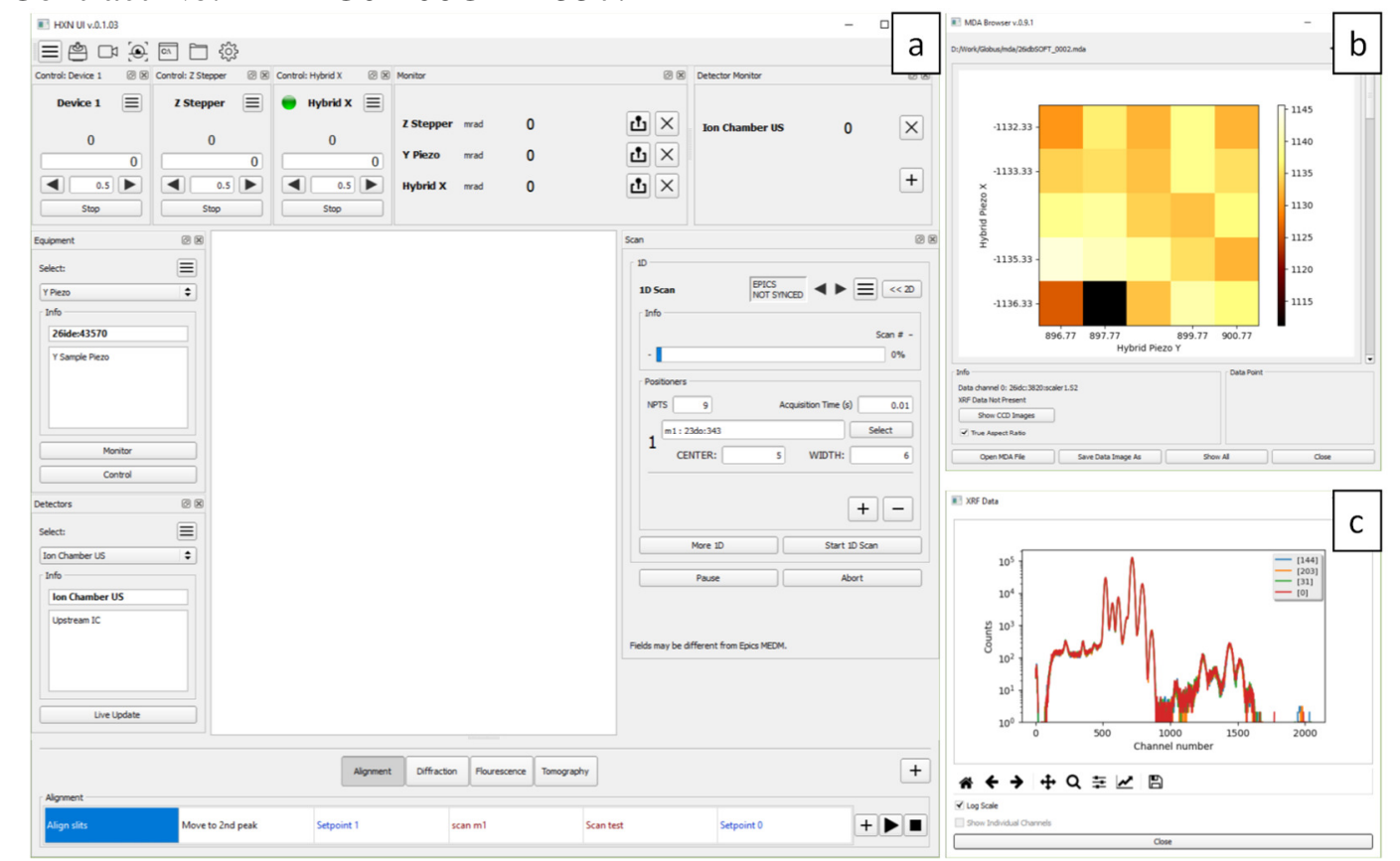

Figure. 1. Main UI is shown in a). Through the toolbar users can load and save UI configuration, bring up Equipment and Detector Browsers located in the left dock area, open Scan Window shown in the right dock area, open Script Window and MDA file browser shown in b) and access Settings. Through Settings panels users can easily add and remove equipment, define detectors, and predefine and save frequently used scan setups. In the top docking area each positioner device can be controlled through Control Widgets or monitored through Monitor Windows. Different Epics Process Variables (PVs) can be monitored in the Detector Monitor Window. Scan progress can be monitored with the progress bar provided in the Scan Window as well as via different detector monitors. Supported detector types which can be monitored are Scaler (b), XRD, and XRF (c) as well as different Epics PVs mentioned above. Task buttons are located at the bottom of the UI and are used to bring up relevant configuration and to display task workflow. 